J. Lake Sci.(湖泊科学), 2008, 20(2): 257-262

http://www.jlakes.org. E-mail: jlakes@niglas.ac.cn

(C)2008 by Journal of Lake Sciences

\title{
微曝气垂直流湿地处理城郊低浓度生活污水模拟实验
}

\author{
柯 凡 ${ }^{1,2}$, 王 䂞 ${ }^{1,2}$, 李海英 ${ }^{1,2}$, 潘继征 ${ }^{1}$, 夏天翔 ${ }^{1,2}$, 李文朝 $1^{* *}$ \\ (1: 中国科学院南京地理与湖泊研究所湖泊与环境国家重点实验室, 南京 210008) \\ (2: 中国科学院研究生院, 北京 100049)
}

摘 要: 本文提出微曝气垂直流湿地的概念, 并通过柱状模型实验模拟研究了其对云南滇池流域城郊低浓度生活污水的处理 效果. 测定了模型的氧利用效率, 最高为 $14.04 \%$. 在高水力负荷 $15.29 \mathrm{~m}^{3} /\left(\mathrm{m}^{2} \cdot \mathrm{d}\right)$ 、气水比 $1: 2$ 的条件下, $\mathrm{BOD}_{5}$ 和 $\mathrm{NH}_{4}-\mathrm{N}$ 去除率 分别为 $74.4 \%$ 和 $63.5 \%$, 出水平均含量分别为 $4.96 \mathrm{mg} / \mathrm{L}$ 和 $2.72 \mathrm{mg} / \mathrm{L}$. 结果说明, 微曝气垂直流湿地工艺能满足低浓度生活污 水高水力负荷的处理要求, 微曝气耗能折价 $0.04-0.05$ 元 $/ \mathrm{m}^{3}$.

关键词: 微曝气垂直流湿地; 生活污水; 模拟实验

\section{Experimental studies on aerated vertical-flow wetland for low concentration domestic wastewater treatment}

\author{
KE Fan ${ }^{1,2}$, WANG Lei ${ }^{1,2}$, LI Haiying ${ }^{1,2}$, PAN Jizheng ${ }^{1}$, XIA Tianxiang ${ }^{1,2}$ \& LI Wenchao ${ }^{1}$ \\ (1: State Key Laboratory of Lake Science and Environment, Nanjing Institute of Geography \& Limnology, Nanjing 210008, P.R.China) \\ (2: Graduate School of the Chinese Academy of Sciences, Beijing 100049, P.R.China)
}

\begin{abstract}
A concept Aerated Vertical-Flow Wetland is introduced in the text, experimental researches through a pillared unit were carried out on the performances of treating domestic wastewater in the rural area of Lake Dianchi valley, Yunnan province. The oxygen transfer efficiency of the experiment device reached $14.04 \%$. And the experiment data showed that, with hydraulic loading as $15.29 \mathrm{~m}^{3} /\left(\mathrm{m}^{2} \cdot \mathrm{d}\right)$ and airflow-waterflow ratio $1: 2$, average $\mathrm{BOD}_{5}$ and $\mathrm{NH}_{4}-\mathrm{N}$ removal respectively reaches $74.4 \%$ and $63.5 \%$. The $\mathrm{BOD}_{5}$ and $\mathrm{NH}_{4}-\mathrm{N}$ values within outflow get to $4.96 \mathrm{mg} / \mathrm{L}$ and $2.72 \mathrm{mg} / \mathrm{L}$. It resulted that, the Aerated Vertical-Flow Wetland Technics was effective and economical for suburb domestic wastewater treatment with high hydraulic loading.

Keywords: Aerated vertical flow wetland; suburb domestic wastewater; simulating experiment
\end{abstract}

构筑湿地(Constructed Wetland)是在自然湿地基础上发展起来的低浓度污水处理生态工程技术，是一 种由人工建造和监督控制的与沼泽地类似的地面, 它利用自然生态系统中的物理、化学和生物的三重协 同作用来实现对污水的净化 ${ }^{[1]}$.

城郊缺乏污水管网，大部分为雨污混流，并且与地面水混合，其主要特点是浓度低、水量大，并且很 难纳人城市污水处理系统. 用构筑湿地处理此类污水有一定的优势，但大部分构造湿地依靠自然复氧和 湿地植物根际的氧化机制, 氧化能力十分有限, 水力负荷一般比较低. 大部分有机污染物通过厌氧过程 去除，存在大气污染问题，并且对氨氮的氧化率较低成为限制脱氮的主要因素，目前在使用构筑湿地来 处理生活污水，其 $\mathrm{BOD}_{5}$ 负荷一般都不超过 $6.2 \mathrm{~g} /\left(\mathrm{m}^{2} \cdot \mathrm{d}\right)^{[2]}$ ，氨氮负荷在 $0.95 \mathrm{~g} /\left(\mathrm{m}^{2} \cdot \mathrm{d}\right)$ 以下 ${ }^{[3]}$; 同时,建设构筑 湿地要占用大量土地，这在土地资源日趋紧缺的今天，尤其在城郊地区是很不现实的，大幅度提高构筑 湿地的负荷可望缓解占地大的问题. 国外已有一些学者采用人工增氧 (Artificial Aeration) 来提高湿地的

* 国家高技术研究计划(863): 河口水质净化技术研究与工程示范(2005AA601010)和缺水地区污水厂尾水复合型湿地深度 净化回用技术(2006AA06Z325)联合资助. 2007-05-17 收稿; 2007-12-04 收修改稿. 柯凡, 男, 1981 生, 硕士研究生, E-mail: cocolor@gmail.com.

** 通讯作者; E-mail: wchli@niglas.ac.cn. 

污水处理能力. Claudiane 等在种植了菖蒲的水平流湿地模型的进水端曝气, 使 $\mathrm{COD}_{\mathrm{Cr}}$ 和总凯氏氮 ( $\mathrm{TKN}$ ) 的去除率达到了 $90 \%$ 以上 ${ }^{[4]}$.

受曝气生物滤池原理的启发, 本文提出一种微曝气垂直流湿地工艺, 在深度 $1.5-2 \mathrm{~m}$ 的碎石床底部铺 设多孔布水管(垂直上升流湿地), 在布水管内安装特制的微量曝气管, 水气同路在碎石床里上升, 在水介 质中通过碎石表面的生物膜进行有氧净化，以达到提高氨氮的硝化率的目的. 在能耗方面微曝气垂直流 湿地只需要 $0.2 \mathrm{~m}$ 左右的水位差来驱动, 但曝气需要消耗一定能量, 设想这种曝气方式的氧气转移率可能 会较高, 因为气泡在上升过程中与碎石表面的生物膜充分接触. 柱状模拟实验旨在通过对微曝气垂直流 湿地氧化净化效率和氧转移率的研究揭示其对城郊低浓度生活污水的处理能力和能耗, 为进一步扩大实 验规模和工程应用提供基础资料.

\section{1 材料与方法}

\section{1 实验装置}

采用 $\phi 200 \mathrm{~mm}$ 的UPVC柱管(图1), 高 $1800 \mathrm{~mm}$. 底部封闭, 并设置进水孔和进气孔, 进气孔内接曝气 砂头. 在 $200 \mathrm{~mm}$ 高度处设置格栅状不锈钢承托架, 在承托架上方填充粒径 $20-40 \mathrm{~mm}$ 碎石(石灰石)作为基 质. 基质层高度 $1300 \mathrm{~mm}$ ，在柱管壁上 $1600 \mathrm{~mm}$ 处设置溢流出水口.

采用连续动态模拟运行方式, 污水取自滇池北岸福保文化城人河排污管口(典型城郊生活污水), 以 小流量水洜连续供水, 经生物接触氧化预处理工艺模型单元后, 自底部进水孔进人本实验装置. 气源由 一台微型空压机提供, 以进气管连接底部进气孔. 在进水管路和进气管路分别设置节流阀和流量计, 以 调控水力负荷和曝气量.

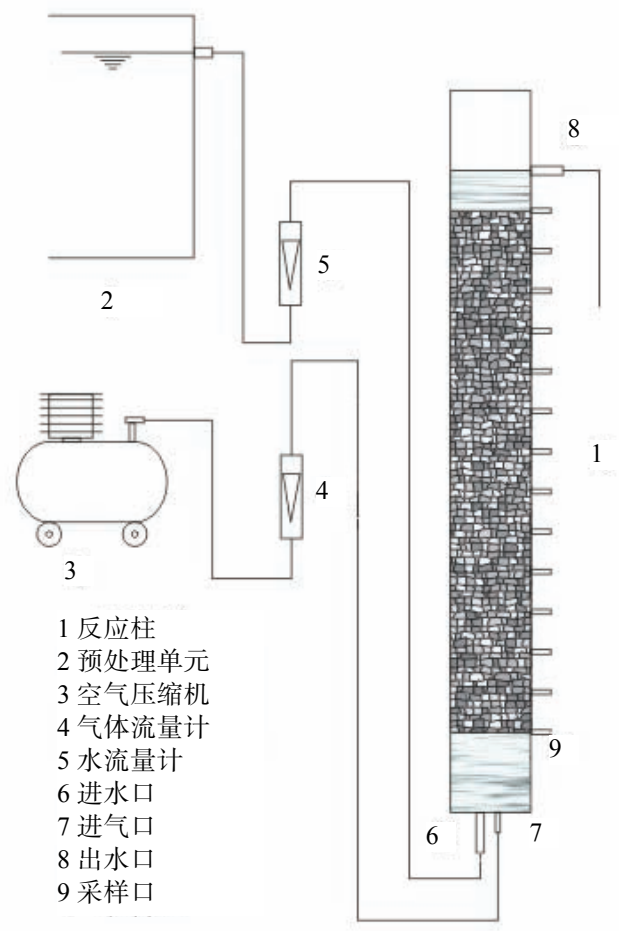

图 1 实验装置图

\section{2 采样与分析}

Fig.1 Experiment units

模型装置于 2006 年 7 月 23 日开始进水挂膜, 2006 年 8 月初挂膜成功并稳定运行到 2007 年 3 月. 模 型进水流量控制为 $20 \mathrm{~L} / \mathrm{h}$, 水力负荷 $15.29 \mathrm{~m}^{3} /\left(\mathrm{m}^{2} \cdot \mathrm{d}\right)$; 采用较低的气水比 $1: 2$. 模型稳定运行一段时间后于 
2006 年 12 月 4 日到 2007 年 3 月 19 日, 隔日采集进水口、出水口水样分析水质指标.

测定的项目有以下水质指标: $\mathrm{DO} 、 \mathrm{BOD}_{5} 、 \mathrm{NH}_{4}-\mathrm{N} 、 \mathrm{NO}_{2}-\mathrm{N} 、 \mathrm{NO}_{3}-\mathrm{N}$, 实验分析方法: DO 用雷磁 JPB-607 溶解氧测定仪测定, $\mathrm{BOD}_{5}$ 用接种稀释法, $\mathrm{NH}_{4}-\mathrm{N}$ 用纳氏试剂法、 $\mathrm{NO}_{2}-\mathrm{N}$ 用 $\mathrm{N}$-(1-荟基)-乙二胺比色法、 $\mathrm{NO}_{3}-\mathrm{N}$ 用酚二磺酸法 ${ }^{[5]}$.

\section{2 结果与讨论}

\section{1 原水水质状况}

进水水质指标见表 1 , 进水的水质波动较大, 主要污染物为有机物和 $\mathrm{NH}_{3}-\mathrm{N}$, 进水平均 $\mathrm{COD}_{\mathrm{Cr}}$ 为 $36.14 \mathrm{mg} / \mathrm{L}$, $\mathrm{BOD}_{5} / \mathrm{COD}_{\mathrm{Cr}}=0.55>0.3$, 说明其可生化性比较好.

表 1 模型实验进水水质统计表

Tab.1 Characteristic of the source water

\begin{tabular}{cccccc}
\hline & 平均值 & 最大值 & 最小值 & 相对偏差 & \multicolumn{2}{c}{ 变异系数 } \\
\hline $\mathrm{pH}$ & 7.87 & 8.21 & 7.56 & 0.17 & 0.02 \\
$\mathrm{COD}_{\mathrm{Cr}}(\mathrm{mg} / \mathrm{L})$ & 36.14 & 75.00 & 4.66 & 16.61 & 0.46 \\
$\mathrm{BOD}_{5}(\mathrm{mg} / \mathrm{L})$ & 19.75 & 35.30 & 5.50 & 7.79 & 0.39 \\
$\mathrm{NH}_{4}-\mathrm{N}(\mathrm{mg} / \mathrm{L})$ & 7.44 & 16.64 & 0.66 & 4.28 & 0.58 \\
$\mathrm{NO}_{2}-\mathrm{N}(\mathrm{mg} / \mathrm{L})$ & 0.73 & 2.96 & 0.00 & 0.86 & 1.17 \\
$\mathrm{NO}_{3}-\mathrm{N}(\mathrm{mg} / \mathrm{L})$ & 0.62 & 2.76 & 0.00 & 0.76 & 1.23 \\
$\mathrm{TN}(\mathrm{mg} / \mathrm{L})$ & 9.52 & 21.81 & 4.21 & 3.94 & 0.41 \\
\hline
\end{tabular}

由于水力负荷达到了 $15.29 \mathrm{~m}^{3} /\left(\mathrm{m}^{2} \cdot \mathrm{d}\right), \mathrm{BOD}_{5}$ 负荷高达 $232.29 \mathrm{~g} /\left(\mathrm{m}^{3} \cdot \mathrm{d}\right)$, 氨氮负荷为 $87.51 \mathrm{~g} /\left(\mathrm{m}^{3} \cdot \mathrm{d}\right)$. 一方 面，高负荷的有机物和氨氮远远超过了一般构筑湿地的正常负荷范围; 另一方面，由于该模型模拟的湿 地系统基质深度为 $1.3 \mathrm{~m}$, 而一般湿地的设计深度仅为 $0.5 \mathrm{~m}^{[6]}$ 到 $1 \mathrm{~m}^{[7-8]}$ 之间, 单纯依靠湿地植物根系泌氧 和大气对复氧作用已经无法满足污染物去除的要求，必须对湿地进行适当的人工增氧. 利用微曝气系统 可以给湿地微生物处理提供好氧环境.

\section{2 氧利用效率与能耗}

微曝气垂直流湿地系统的主要动力消耗集中在曝气系统上, 曝气系统的能耗决定于氧的利用效率. 通过氧的转移率可以计算出不同曝气强度下的氧利用效率 $E_{A}$, 即曝气过程中氧气由气相转移至液相的百 分率, 它是表征设备传质功效的另一重要参数.

根据本研究中的模型装置实验结果和云南滇池地区有关地理参数, 计算得到的氧传递速率常数和氧 利用效率见表 2 .

表 2 空白水柱和填充砾石基质水柱中氧利用效率

Tab.2 Oxygen transfer efficiency in blank and limestone substrate

\begin{tabular}{ccccccc}
\hline 项 目 & \multicolumn{3}{c}{ 空 白 水 柱 } & \multicolumn{3}{c}{ 填充砾石基质水柱 } \\
\hline 曝气量 $(\mathrm{ml} / \mathrm{min})$ & 100 & 200 & 400 & 100 & 200 & 400 \\
$T\left({ }^{\circ} \mathrm{C}\right)$ & 25.4 & 21.2 & 25.4 & 22.8 & 13.8 & 14.8 \\
$c_{s}(\mathrm{mg} / \mathrm{L})$ & 6.80 & 7.36 & 6.80 & 7.15 & 8.59 & 8.40 \\
$K_{L a}(1 / \mathrm{h})$ & 0.2575 & 0.4739 & 0.7684 & 1.3789 & 2.6784 & 4.6893 \\
相关系数 $\left(R^{2}\right)$ & 0.9891 & 0.9962 & 0.9992 & 0.972 & 0.9659 & 0.9725 \\
$K_{L a 20}(\mathrm{~h})$ & 0.2265 & 0.4606 & 0.6760 & 1.2903 & 3.1027 & 5.3048 \\
氧利用效率 $E_{A}(\%)$ & 2.05 & 2.08 & 1.53 & 11.68 & 14.04 & 12.01 \\
\hline
\end{tabular}


从表 2 中的氧利用效率 $E_{A}$ 一栏看出, 填充了砾石基质的水柱中氧利用效率大于 $10 \%$, 而未填充基质的 空白模型其利用氧利用效率仅为 $2 \%$ 左右. 砾石基质水柱中三种曝气量的数据对比显示, 在 $200 \mathrm{ml} / \mathrm{min}$ 的曝 气强度下, 氧的利用效率最高达到了 $14.04 \%$. 氧利用率实验是在挂膜前的模型中进行的, 实际模型挂膜后 的氧利用率应大于上述值.

模型实验中采用了 $1: 2$ 的气水比，根据实验得出的氧利用率 $E_{A}$ 可以计算实际工程应用中的曝气能耗. 参见表 3 .

表 3 曝气能耗计算表

Tab.3 Power consumption of areation

\begin{tabular}{|c|c|c|c|c|c|}
\hline \multirow{2}{*}{ 型号 } & \multicolumn{3}{|c|}{ 常用曝气机械参数 } & \multicolumn{2}{|c|}{ 单方水处理能耗计算 } \\
\hline & 压力 $(\mathrm{kPa})$ & 供气量 $\left(\mathrm{m}^{3} / \mathrm{min}\right)$ & 功率 $(\mathrm{kW})$ & 单方水供气量 $\left(\mathrm{m}^{3}\right)$ & 能耗(kW·h) \\
\hline $\begin{array}{l}\text { BH50 罗茨风机 } \\
\end{array}$ & 20 & 1.33 & 1.1 & 0.5 & 0.0505 \\
\hline BH125A 罗茨风机 & 20 & 10.8 & 7.5 & 0.5 & 0.0424 \\
\hline
\end{tabular}

电费按每千瓦时 1 元计算, 处理一方水需要的曝气费用为 $0.04-0.05$ 元. 按这样计算, 一个处理 10000 $\mathrm{m}^{3} / \mathrm{d}$ 水的微曝气垂直流湿地每天用于曝气的费用为 424 元.

\section{3 有机物的降解}

一般构筑湿地处理污水时, 湿生植物通过通气组织的运输, 将氧气输送到根区，在植物根须周围微 环境中依次出现好氧区、兼氧区和厌氧区, 为好氧微生物和厌氧微生物大量存在提供了条件, 废水中大 部分有机物在好氧区内被好氧微生物氧化分解 ${ }^{[9]}$.

本研究在深度 $1.3 \mathrm{~m}$ 的填料层底部进行微量曝气, 使得整个填料柱中的溶解氧浓度提高, 模型出水 的溶解氧平均值达到了 $3.65 \mathrm{mg} / \mathrm{L}$; 同时从底部进人的污水正好提供了丰富的有机物质, 有利于好氧异养 微生物的生长形成生物膜. 进水中不溶性的有机物通过基质的吸附、过滤等物理作用被截留下来, 并且 在微生物的作用下进一步分解, 溶解性的有机物在经过生物膜表面时被吸附然后被微生物利用 ${ }^{[10]}$.

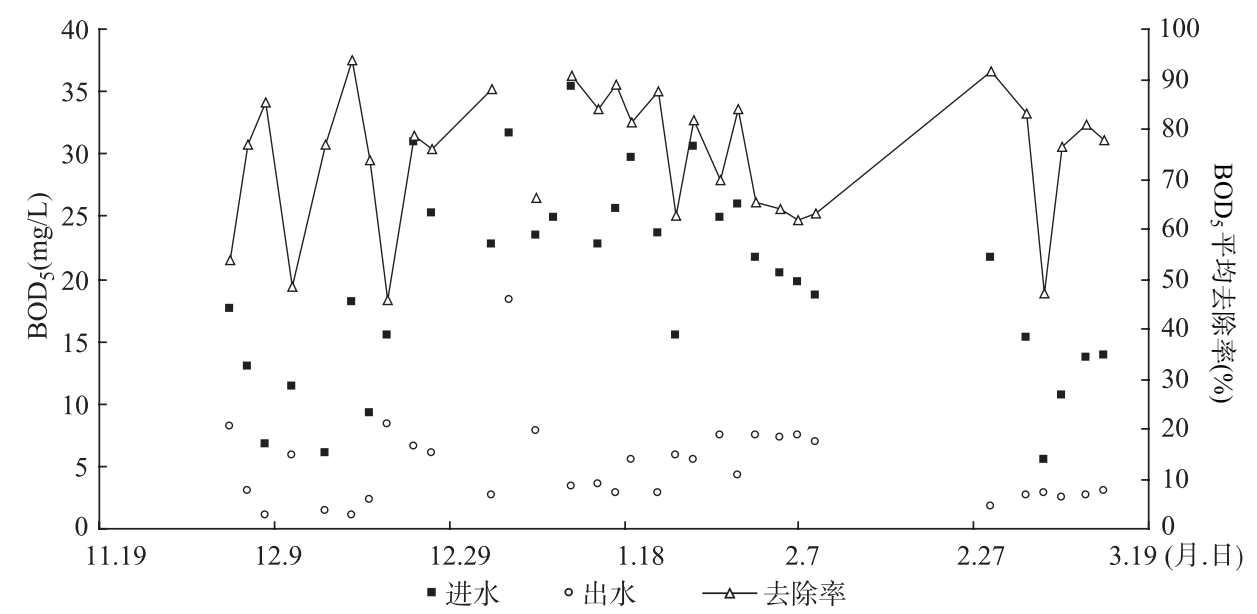

图 $2 \mathrm{BOD}_{5}$ 去除效果

Fig. $2 \mathrm{BOD}_{5}$ removal

从图 2 中可以看到，出水的 $\mathrm{BOD}_{5}$ 基本低于 $10 \mathrm{mg} / \mathrm{L}$, 其平均值为 $4.96 \mathrm{mg} / \mathrm{L}$, 平均去除率达 $74.4 \%$. 其 他研究者在垂直流湿地中也仅取得了 $78.7 \%$ 的 $\mathrm{BOD}_{5}$ 去除率，但水力负荷仅为 $0.2 \mathrm{~m}^{3} /\left(\mathrm{m}^{2} \cdot \mathrm{d}\right)^{[11]}$. 孙广智 ${ }^{[12]}$ 等在伯明翰进行的下行流芦苇床实验中, 在水力负荷为 $0.86 \mathrm{~m}^{3} /\left(\mathrm{m}^{2} \cdot \mathrm{d}\right)$ 的情况下, 进水经过三级芦苇床处 理后获得 $\mathrm{BOD}_{5}$ 去除率为 $74.3 \%$, 与本研究中的结果接近.

出水中的的 $\mathrm{COD}_{\mathrm{Cr}}$ 平均值为 $24.40 \mathrm{mg} / \mathrm{L}$, 此时 $\mathrm{BOD}_{5} / \mathrm{COD}_{\mathrm{Cr}}=20.33 \%$, 即出水可生化性比较差, 说明 
污水在模型中已经被微生物充分分解, 生物可降解的有机物浓度已经达到了相当低的水平.

\section{4 氮的降解与转化}

湿地中氮的去除最主要是通过微生物的硝化一反硝化作用来实现. 其中硝化细菌(包括亚硝酸菌和 硝酸菌)在好氧条件下将氨氮氧化成硝酸盐, 反硝化菌在厌氧条件下利用硝酸盐作为电子受体, 将其还原 为 $\mathrm{N}_{2}$ 或者 $\mathrm{N}_{2} \mathrm{O}^{[13]}$. 污水中 $\mathrm{NH}_{4}-\mathrm{N}$ 的去除率与根区的硝化细菌数量的相关性极显著 ${ }^{[14-15]}$.

在本模型实验中, 进、出水中无机氮的组成及变化见图 3, 进水中以氨态氮占优, 平均为总氮 $(\mathrm{TN})$ 含 量的 $74.2 \%$, 最高为 $99 \%$. 经过微曝气垂直流湿地模型处理后, 大部分氨氮被氧化成硝氮, 氨氮的平均去 除率为 $63.5 \%$. 出水中 $\mathrm{NO}_{3}-\mathrm{N}$ 平均占三态氮总量的 $65.6 \%$, 残余氨氮平均浓度为 $2.72 \mathrm{mg} / \mathrm{L}$. 朱夕珍等在美 人蕉模拟垂直流人工湿地中去除氨氮 $83.4 \%-45.3 \%$, 其水力负荷为 $0.07-0.18 \mathrm{~m}^{3} /\left(\mathrm{m}^{2} \cdot \mathrm{d}\right)$; 崔理华利用煤炭 - 草碳基质垂直流人工湿地系统在水力负荷为 $0.54-0.64 \mathrm{~m}^{3} /\left(\mathrm{m}^{2} \cdot \mathrm{d}\right)$ 时取得的氨氮去除率为 $75 \%-85 \%$. 单从 氨氮去除率来看, 本研究与其他研究的结果比较接近; 但由于本研究的水力负荷高达 $15.29 \mathrm{~m}^{3} /\left(\mathrm{m}^{2} \cdot \mathrm{d}\right)$, 可 见微曝气垂直流湿地更适合大流量, 低浓度的污水处理, 而处理效果和其他的湿地系统接近.
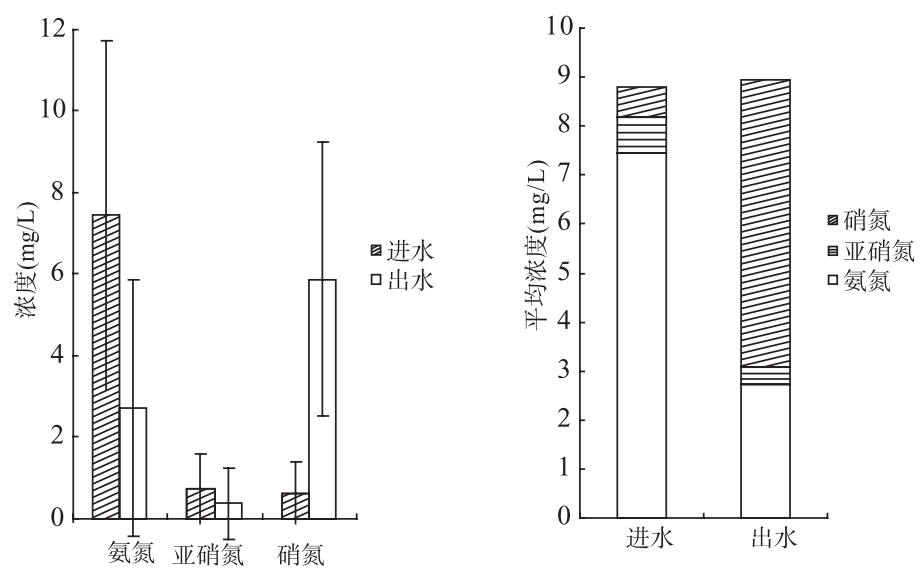

图 3 进出水中的三态氮浓度比较

Fig. 3 Comparisons between inflow and outflow on 3 forms of nitrogen

另一方面, 对比图 3 中右图三态氮的总和(即柱状图高度), 可以发现出水的 $\mathrm{NH}_{4}-\mathrm{N} 、 \mathrm{NO}_{2}-\mathrm{N} 、 \mathrm{NO}_{3}-\mathrm{N}$ 三 种形态氮数值之和要高于进水, 说明有机氮在模型中进行了转化, 有机大分子形态分解转化成三态氮, 这 符合氮的降解规律. 同时还说明, 微曝气垂直流湿地中的氧化环境不利于反硝化脱氮, 出现了硝氮的积累.

氨氮的去除速率常数 $\mathrm{K}$ 可以从一级动力学方程中得到:

$$
C_{0}=C_{i} \mathrm{e}^{-K A / Q}
$$

式中: $C_{0}$ 为构筑湿地出口 $\mathrm{NH}_{4}-\mathrm{N}$ 的值 $(\mathrm{mg} / \mathrm{L}) ; C_{i}$ 为构筑湿地进口 $\mathrm{NH}_{4}-\mathrm{N}$ 的值 $(\mathrm{mg} / \mathrm{L}) ; A$ 为构筑湿地表面积 $\left(\mathrm{m}^{2}\right) ; Q$ 为日流量 $\left(\mathrm{m}^{3} / \mathrm{d}\right) ; K$ 为去除速率常数 $(\mathrm{m} / \mathrm{d})$.

表 $4 \mathrm{NH}_{4}-\mathrm{N}$ 的去除速率常数

Tab.4 Coefficient of $\mathrm{NH}_{4}-\mathrm{N}$ remove rate

\begin{tabular}{|c|c|c|c|c|c|}
\hline & 平均值 & 最大值 & 最小值 & 方 差 & 变异系数 $C V$ \\
\hline 进 水 $(\mathrm{mg} / \mathrm{L})$ & 7.44 & 16.64 & 0.66 & 4.28 & 0.58 \\
\hline 出 水 $(\mathrm{mg} / \mathrm{L})$ & 2.72 & 12.59 & 0.00 & 3.14 & 1.16 \\
\hline 去除速率常数 $K(\mathrm{~m} / \mathrm{d})$ & 17.01 & 46.88 & -13.54 & 15.10 & 0.89 \\
\hline
\end{tabular}

抚仙湖窑泥沟构筑湿地中 $\mathrm{NH}_{4}-\mathrm{N}$ 的去除速率常数为 $0.34 \mathrm{~m} / \mathrm{d}^{[16]}$, 在武汉东湖测知的 $\mathrm{NH}_{4}-\mathrm{N}$ 去除速率 常数为 $0.80 \mathrm{~m} / \mathrm{d}^{[11]}$. 表 4 中的氨氮去除速率常数平均值为 $17 \mathrm{~m} / \mathrm{d}$, 说明微曝气系统对垂直流湿地的氨氮去 
除能力有极大提升, 可以满足高负荷的氨氮去除要求.

\subsection{SS 和 P 的去除}

垂直流湿地对 SS 的去除机理和生物滤池相类似, 主要通过基质的过滤、吸附作用而被截留下来, 实 验中获得了 $70.9 \%$ 平均去除率，出水中的平均浓度低达 $1.55 \mathrm{mg} / \mathrm{L}$. 同时 SS 的截流也会带来填料堵塞问题, 这可以通过预处理降低进水浓度来预防, 本实验中进水 $\mathrm{SS}$ 平均浓度仅为 $5.37 \mathrm{mg} / \mathrm{L}$.

$\mathrm{P}$ 的去除主要通过基质的过滤吸附、化学沉淀作用、植物的吸收、微生物的合成代谢等途径, 在本 实验模型中去除能力十分有限, TP 在出水中的平均浓度为 $0.62 \mathrm{mg} / \mathrm{L}$, 去除率为 $17.7 \%$.

\section{3 结论}

(1)填充砾石基质的微曝气垂直流湿地模拟实验柱在未挂膜的情况下, 微量曝气的氧利用效率在 $11 \%-14 \%$ 之间, 在 $200 \mathrm{ml} / \mathrm{min}$ 的曝气强度下达到最大值 $14.04 \%$, 是同等曝气条件下无填料水柱中氧利用 效率的 7 倍.

(2)在高水力负荷条件下, 模型能够有效去除低浓度生活污水中的 $\mathrm{BOD}_{5}$ 和 $\mathrm{NH}_{4}-\mathrm{N}$, 出水水质已经达 到污水综合排放一级标准(GB18918-2002), 说明微曝气垂直流湿地能达到原 $\mathrm{BOD}_{5}$ 氧化和氨氮硝化的设 计要求, 并且其氧化处理能力远高于普通的构造湿地和自然湿地系统.

(3)微曝气垂直流湿地模型属于加强型氧化单元，其出水含有一定浓度的硝态氮，可与后续工艺单元 配合以取得更好的总氮去除效果.

(4)采用微曝气垂直流湿地高水力负荷处理低浓度污水是一个经济有效的工艺方案, 处理每方水的曝 气费用为 $0.04-0.05$ 元.

\section{4 参考文献}

[1] Tennessee Valley Authority. River basin operations for treatment of municipal wastewater. Monitory Report For the period: March 1988 to October 1989, 1990.

[2] 张荣社, 周 琪, 张 建等. 潜流构造湿地去除农田排水中氮的研究. 环境科学, 2003, 24(1): 113-116.

[3] 刘剑粀, 丘昌强, 陈珠金等. 复合生态系统工程中高效去除磷、氮植被植物的笁选研究. 水生生物学报, 1998, 22(1): 1-8.

[4] Claudiane OP, Florent Ch, Yves Co et al. Artificial aeration to increase pollutant removal efficiency of constructed wetlands in cold climate. Ecological Engineering, 2006, 27: 258-264.

[5] 国家环境保护总局. 水和废水监测分析方法(第4版). 北京: 中国环境科学出版社, 1998.

[6] 吴振斌, 詹德吴, 张 晟等. 复合垂直流构建湿地的设计方法及净化效果. 武汉大学学报(工学版), 2003, 36(1): 12-16.

[7] 雷志洪, 戴知广, 陈志诚等. 高效复合垂直流构筑湿地系统处理效果与污水回用工程. 给水排水, 2002, 28(9): 22-24.

[8] 迟延智, 陈风伦. 构筑湿地处理污水的实践. 中国给水排水, 2003, 19(4): 82-83.

[9] 吴献花, 侯长定, 王 林等. 构筑湿地处理污水的机理. 玉溪师范学院学报, 2002, 18(1): 103-105.

[10] 吴晓磊. 构筑湿地废水处理机理. 环境科学, 1995, 16(3): 83-86.

[11] 吴振斌, 成水平, 贺 锋等. 垂直流人工湿地的设计及净化功能初探. 应用生态学报, 2002, 13(6): 715-718.

[12] 孙广智, Gray KR, Biddleston AJ. 下行流芦苇床污水处理试验研究与设计方程. 中国给水排水, 1997, 13: 4-6.

[13] CP Leslie Grady Jr, Glen T Daigger, Henry C Lim. Biological wastewater treatment. New York: CRC Press, 1999: $135-143$.

[14] 李科得, 胡正嘉. 芦苇床系统净化污水的机理. 中国环境科学, 1995, 15(2): 140-144.

[15] 李科得, 胡正嘉. 人工模拟芦苇床系统处理污水的效能. 华中农业大学学报, 1994, 13(5): 511-517.

[16] 陈源高, 李文朝, 李荫罕等. 云南抚仙湖窑泥沟复合湿地的除氮效果. 湖泊科学, 2004, 16(4): 331-336. 